\title{
One swallow does not a summer make but many swallows do: accumulating clinical evidence for nearly-eliminated peri-procedural and 30-day complications with mesh- covered stents transforms the carotid revascularisation field
}

\author{
Piotr Musiałek ${ }^{1}$, L. Nelson Hopkins², Adnan H. Siddiqui² \\ 1Department of Cardiac and Vascular Diseases, Jagiellonian University, School of Medicine, John Paul II Hospital, Krakow, Poland \\ ${ }^{2}$ Departments of Neurosurgery and Radiology, School of Medicine and Biomedical Sciences, University at Buffalo, State University \\ of New York, Jacobs Institute, Gates Vascular Institute Kaleida Health, Buffalo, New York, USA
}

Adv Interv Cardiol 2017; 13, 2 (48): 95-106

DOI: https://doi.org/10.5114/pwki.2017.69012

\begin{abstract}
Atherosclerotic carotid artery stenosis (CS) continues to be a common cause of acute ischaemic stroke. Optimised medical therapy (OMT), the first-line treatment modality in CS, may reduce or delay - but it does not abolish - CS-related strokes. As per current AHA/ASA and ESC/ESVS/ESO guidelines, carotid artery stenting (CAS) is a less-invasive alternative to carotid endarterectomy (CEA) for CS revascularisation in primary and secondary stroke prevention.

Ten-year follow-up from the CREST trial in patients with symptomatic and asymptomatic CS confirmed equipoise of CAS and CEA in the primary endpoint. Nevertheless CAS - using a widely open-cell, first-generation stent and first-generation (distal/filter) neuroprotection - has been criticised for its relative excess of (mostly minor) strokes by 30 days, a significant proportion of which were post-procedural.

Atherosclerotic plaque protrusion through conventional carotid stent struts, confirmed on intravascular imaging, has been implicated as a leading mechanism of the relative excess of strokes with CAS vs. CEA, including delayed strokes with CAS. Different designs of mesh-covered carotid stents have been developed to prevent plaque prolapse. Several multi-centre/multi-specialty clinical studies with CGurad MicroNet-Covered Embolic Prevention Stent System (EPS) and RoadSaver/Casper were recently published and included routine DW-MRI cerebral imaging peri-procedurally and at 30 days (CGuard EPS).

Data from more than 550 patients in mesh-covered carotid stent clinical studies to-date show an overall 30-day complication rate of $\sim 1 \%$ with near-elimination of post-procedural events. While more (and long-term) evidence is still anticipated, these results - taken together with optimised intra-procedural neuroprotection in CAS (increased use of proximal systems including trans-carotid dynamic flow reversal) and the positive 12-month mesh-covered stent data reports in 2017 - are transforming the carotid revascularisation field today.

Establishing effective algorithms to identify the asymptomatic subjects at stroke risk despite OMT, and large-scale studies with mesh-covered stents including long-term clinical and duplex ultrasound outcomes, are the next major goals.
\end{abstract}

Key words: carotid artery stenting, mesh, stroke, endarterectomy, neuroprotection.

\section{Atherosclerotic carotid stenosis and ischaemic stroke}

Acute ischaemic stroke is the leading cause of premature mortality and morbidity worldwide for both men and women [1, 2]. Atherosclerotic carotid artery stenosis continues to be a major cause of acute ischaemic strokes [1-4]. Accordingly, patients with symptomatic carotid stenosis account for up to $\sim 30-60 \%$ of subjects enrolled in contemporary clinical trials of carotid revascularisation [5-8]. Some of these patients develop symptoms of carotid stenosis-associated cerebral ischaemia despite optimised medical therapy (OMT) [5, 9-12], consistent with the concept that OMT may reduce or delay [10, 13], but does not abolish, the stroke risk in relation to carotid atherosclerosis $[9,10,12]$. Thus, carotid artery stenosis, accounting for $\sim 15-20 \%$ of acute ischaemic strokes to-

\section{Corresponding author:}

Piotr Musiatek MD, DPhil (Oxford), FESC, Department of Cardiac and Vascular Diseases, Jagiellonian University, School of Medicine, John Paul II Hospital, 80 Pradnicka St, 31-202 Krakow, Poland, fax: +48 12614 33 32, e-mail: pmusialek@szpitaljp2.krakow.pl

Received: 3.07.2017, accepted: 10.07.2017. 
day [1-4], continues to be an important and modifiable risk factor for ischaemic stroke [1].

Stroke in relation to atherosclerotic carotid artery stenosis occurs through progressive (usually in a non-linear fashion) narrowing and resultant hypoperfusion in the ipsilateral hemisphere secondary to an incompetent circle of Willis or plaque rupture or erosion and consequent thrombus formation. However, most often the stroke mechanism is embolic rather than haemodynamic [14, 15]. Contemporary vascular clinic data indicate, in medically-treated patients with a significant carotid stenosis, a stroke risk of 2\% per year [9]. Because the yearly stroke risk is cumulative, such a seemingly "low" (to some) risk indicates, for instance, for a 50-year old medically-treated person with asymptomatic carotid stenosis, an absolute stroke risk of $\sim 40 \%$ by age 70 . This is not negligible to the patient and family. And it should not be neglected by the physician who can either significantly reduce this substantial stroke risk with safe carotid revascularization, or refer the patient to another physician/centre where carotid revascularization with a minimal procedural risk can be performed.

According to current guidelines $[16,17]$ and clinical practice [2-7, 18-20], the endovascular route of carotid revascularisation (carotid artery stenting - CAS) is an alternative to carotid endarterectomy (CEA) in symptomatic and asymptomatic patients with atherosclerotic carotid stenosis.

\section{CREST and its impact on carotid artery stenting}

Recent 10-year follow-up data from the Carotid Revascularisation Endarterectomy versus Stenting Trial (CREST) demonstrated - in 2502 patients with symptomatic or asymptomatic carotid stenosis randomised on a $1: 1$ basis to transfemoral CAS or CEA - no difference in the rate of the extended study primary endpoint (composite of periproedural stroke up to 30 days, and any myocardial infarction (MI) death, or any subsequent ipsilateral stroke - hazard ratio $1.10,95 \%$ confidence interval (CI): 0.83-1.44) [21]. There was no difference between CAS and CEA in stroke rate after 30 days, and no difference - in any study endpoint - for symptomatic vs. asymptomatic patients [21].

For many evidence-oriented physicians, large-scale level 1 evidence with long-term follow-up settles firmly the role of CAS in carotid revascularisation for primary and secondary stroke prevention in relation to atherosclerotic carotid artery stenosis. For others, concerned with the equipoise of the two procedures in CREST [22], release of 10-year CREST data has been the occasion to attempt to reignite the decade-long debate [23] on the relative importance of individual composites of the CREST primary endpoint [24]. This is because in the absence of any significant differences in major stroke or death between the two procedures, in CREST there was a relative excess of
MI with CEA and a relative excess of (minor and occurring up to 30 days post procedure) strokes with CAS [21, 24]. Consistent with the signal from CREST, recent meta-analysis of over 6500 patients from five trials with a mean follow-up of 5.3 years confirmed that CAS, using first-generation carotid stents and mainly distal (filter) neuroprotection, was indeed associated with a relative excess of (mostly minor and occurring mostly peri-procedurally and up to 30 days) strokes (odds ratio $(\mathrm{OR})=1.50,95 \% \mathrm{Cl}$ : 1.22-1.84) [25]. In contrast, the risk of periprocedural MI was significantly lower with CAS than with CEA (OR $=0.45,95 \% \mathrm{Cl}: 0.27-0.75)$ and the composite of death, stroke, MI, or cranial nerve palsy by 30 days was significantly lower with CAS (OR $=0.75,95 \% \mathrm{Cl}$ : 0.60-0.93) [25].

Follow-up of the CREST patients clearly demonstrated that both periprocedural MI (seen more frequently with CEA) and procedure-related stroke (which occurred more frequently in the CAS arm) were prognostically important. The risk of death at 4 years was higher after peri-procedural stroke, hazard ratio $2.78 ; 95 \% \mathrm{Cl}$ : 1.63-4.76 [26]. Similarly, patients with $\mathrm{MI}$ showed a significantly higher 4-year mortality, hazard ratio 3.40, 95\% Cl: 1.67-6.92; an effect that remained significant after adjustment for baseline risk factors $[27,28]$. In addition, using different data-sets, arguments have been raised that "real-life" outcomes may be worse-than-CREST for CAS [29] as well as for CEA [30]. However, as procedural strategies and techniques have significantly evolved in the last decade, paralleled by a further increase in the (endovascular in particular) operator experience, the discussion of historical data may be chiefly of a historical value today.

\section{Evolution of carotid revascularisation strategies after CREST}

Fundamental changes in carotid revascularisation equipment and strategies after CREST include: (1) substantial improvements in intra-procedural brain protection in CAS (more effective distal protection devices and increased understanding of the efficacy and role for proximal protection, new proximal protection systems that avoid the aortic arch), (2) novel carotid stent designs (mesh-covered stents to minimise plaque prolapse and embolisation during and after the procedure), (3) tailoring the neuroprotection device and stent type to the patient's symptomatic status and plaque type, (4) better case selection for avoiding high-risk CAS, which may be lower risk for CEA such as severe arch (N.B. trans-carotid CAS overcomes the arch problem) and proximal vessel disease including tortuosity as well as lesional angulation and length or highly calcific lesions, and avoiding patients with potentially severe consequences of the carotid baroreflex activation with CAS (such as concomitant severe aortic valve disease), and (5) increased understanding of the importance of coronary risk evaluation and work-up to minimise the risk of peri-procedural MI. 
A substantial body of clinical evidence indicates that proximal neuroprotection [31-34] including dynamic flow reversal $[35,36]$ can minimise the risk of intra-procedural cerebral embolisation [37-43]. According to "tailored CAS" algorithms [5, 34, 37, 44, 45], proximal neuroprotection is preferred in endovascular management of symptomatic and high-risk asymptomatic lesions. Indeed, compared with use of a filter, proximal neuroprotection significantly reduced the number of microembolic signals (MES) $(p<0.0001)$ during lesion crossing (mean 2; interquartile range (IQR): 0 to 4 vs. 18; IQR: 11 to 30), stent crossing (0; IQR: 0 to 1 vs. 23; IQR: 11 to 34), stent deployment (0; IQR: 0 to 1 vs. 30; IQR: 9 to 35), stent dilation (0; IQR: 0 to 1 vs. 16; IQR: 8 to 30), and the total MES number (16; IQR: 7 to 36 vs. 93; IQR: 59 to 136) [39]. Moreover, the proportion of patients with any MES was profoundly lower with proximal device versus filter in phases 3 to 5 ( $27 \%$ vs. $100 \% ; p<0.0001$ ) [39].

It is important to note that the endovascular arm of CREST employed an extremely open-cell carotid stent $[21,25]$ (individual cell area of $11.48 \mathrm{~mm}^{2}$ - the largest cell size among commonly used conventional carotid stents [46]). The advantage of an open-cell design is that it conforms to tortuous anatomy well. However, the concern over large cell size, as is the case with most open cell designs, is that post stenting the plaque has greater unconstrained areas through which it can extrude through the stent tines and embolise intracranially causing post stenting cerebral infarct that can manifest as clinical stroke. Data from registries that used several conventional carotid stent types indicate an increase in the risk of neurological events with an increase in cell area [46]. This was confirmed by analysis from Stent-Protected Angioplasty versus Carotid Endarterectomy in Symptomatic Patients (SPACE-1) prospective trial data, focused on the impact of the stent design on peri-interventional complication rate. In SPACE-1, the stroke risk was significantly higher in patients treated with an open cell stent $(11.0 \%, 95 \% \mathrm{Cl}: 6.2-17.8 \%)$ in comparison to those in whom a closed cell stent was used $(5.7 \%, 95 \% \mathrm{Cl}$ : $3.7-8.3 \%, p<0.05)$, and the stent design appeared to have a more important impact on CAS neurological complications than the protection device [47]. Similarly, analysis of adverse outcome predictors in 828 patients randomised to the CAS arm of the International Carotid Stenting Study (ICSS), demonstrated that use of an opencell stent conferred a statistically significant two-fold higher risk of stroke by 30 days than use of a closed-cell stent (relative risk $(\mathrm{RR})=1.92,95 \% \mathrm{Cl}$ : 1.11-3.33) [48]. There are theoretical grounds and there is some moderate-scale evidence that hybrid stents, which combine the open-cell design advantage of high conformability (proximal and distal stent section) with the closed-cell design of the mid-section of the stent aimed to cover the plague, may be non-inferior to closed-cell stents [49].
Recent individual patient data analysis of 1604 patients who underwent neuroprotected CAS in the European Registry of Carotid Artery Stenting (ERCAS) demonstrated that the use of an open-cell design stent with a free cell area $>7.5 \mathrm{~mm}^{2}$ was associated with a significantly increased 30-day stroke risk [50], highlighting the importance of carotid stent design in minimising neurological complications following CAS.

Not only was neuroprotection in the CAS arm of CREST limited to a distal device that needs to be delivered through the lesion prior to establishing temporary brain protection $[24,39,51]$, but also the device was a first-generation filter subsequently implicated to provide a limited, suboptimal neuroprotective effect [38, 52, 53]. Moreover, there has been increasing understanding that filters may not to be applicable to all patients and lesions when an endovascular revascularisation route is used as the primary strategy [5, 36-38, 42, 44]. A recent meta-analysis of 8 studies (357 patients) compared the results of distal filter cerebral protection vs. proximal balloon occlusion in preventing embolisation during CAS as evaluated by diffusion-weighted magnetic resonance imaging of the brain (DW-MRI) [40]. The incidence of new ischaemic lesions after CAS detected by DW-MRI was significantly lower in the proximal protection group (effect size: -0.43 ; $95 \% \mathrm{Cl}:-0.84$ to -0.02 ) [40], indicating an important role for proximal neuroprotection in increasing the procedural (but not post-procedural) safety of CAS. Indeed, the role of optimised neuroprotection to achieve a safe CAS procedure is increasingly understood and has impacted clinical practice, with proximal embolic protection used in $\sim 50 \%$ or greater proportion of CAS procedures in the leading CAS centres today [5, 7, 37, 43, 44].

CREST $[24,26]$ confirmed observations from other large clinical studies using conventional carotid stents [54] that a significant proportion of strokes seen by 30 days occurred after completion of carotid revascularisation procedure, when the temporary embolic protection device was no longer in place. Indeed, with conventional open- or closed-cell carotid stents, $40-80 \%$ of all adverse neurological events by 30 days are post-procedural $[26,46,54]$.

Serial DW-MRI cerebral imaging demonstrated post-procedural cerebral embolisation after CAS using conventional stents [55]. In transfemoral CAS, open-cell stent design was shown to be associated with a 2-fold increase $(p<0.001)$ in the risk of periprocedural cerebral embolisation [38], consistent with the clinical evidence for a 2-fold increase in stroke up 30 days with opencell vs. closed-cell conventional carotid stents [47, 48]. The post-procedural cerebral embolisation $[38,55]$ and post-procedural neurological complications [26, 46-48, 54] have been linked to the atherosclerotic plaque protrusion through the stent struts [56-59]. Atherosclerotic plaque prolapse occurs (depending on the visualisation technique such as angiography, intravascular ultrasound, 
or optical coherence tomography) in up to $30-60 \%$ of conventional carotid stents and it may be reduced but not eliminated with the conventional closed-cell stent designs [56-59]. Recent systematic evaluation of a large patient series (328 consecutive patients, $48 \%$ symptomatic) with CAS accompanied by routine intravascular ultrasound imaging confirmed a significant increase in plaque prolapse susceptibility with the unstable carotid plaque and open-cell stent use [8]. Plaque protrusion was strongly associated not only with new ischaemic lesions on DW-MRI but also with the clinical manifestation of perioperative ischaemic stroke $(p<0.0001)$, indicating the necessity to prevent plaque prolapse [8]. Mesh-covered carotid stents, designed to prevent plaque prolapse, have been developed and implemented in the clinical practice of CAS, leading to an increasing body of clinical evidence in the public domain today [5-7, 60-63].

Following CREST, surgical strategies have been evolving too. Today, in many centres elective CEA is preceded by clinical and/or imaging screening for relevant coronary artery disease and is performed under local anaesthesia either as a rule [64] or in increased-coronary-risk patients [65] to minimise the risk of CEA-associated MI. Surgeons are increasingly adopting the endovascular route to resolve important vascular pathologies such as aortic aneurysms, aorto-iliac, ilio-femoral, and below-theknee atherosclerotic disease leading to limb ischaemia, and the endovascular route, to many today, is natural in every-day practice. In the carotid territory, it is most recently exemplified by an increasing adoption of CAS using surgical access via the common carotid artery and a robust ('dynamic') flow reversal system for temporary brain protection (trans-carotid arterial revascularisation - TCAR) [35, 36, 38, 42, 66].

\section{Mesh-covered carotid stents to minimise intra-procedural and prevent post- procedural cerebral embolisation: designs and bench data}

Minimisation of intra-procedural [38, 40, 43, 67] and prevention of post-procedural [55] cerebral embolism that, in a proportion of patients, is manifested as clinical strokes $[26,46,54]$ have become fundamental to CAS as a treatment strategy. For CAS to continue as a viable treatment modality, the surgical community postulated the necessity to develop better stents $[68,69]$ and to increase the use of proximal rather than distal protection [68].

The use of ultra-closed cell stent systems (N.B. the ultra-closed cell design is achieved with the technology of covering the nitinol frame with a mesh that can be made of different materials), on the other hand, can not only further reduce the risk of intra-procedural neurological complications but also, by preventing plaque protrusion through stent struts, can eliminate the post-procedural cerebral embolisation as manifested on routine DW-MRI
[60]. This strategy has been termed intra- and post-procedural (sustained) "embolic prevention" [5, 10]. Sustained embolic prevention is thus complementary to the classic intra-procedural "embolic protection" using the proximal (flow clamping or reversal) or distal (filter) temporary devices [10].

As of today, three different mesh-covered stents have been developed, including (1) RoadSaver/Casper [70-72] (Terumo/Microvention), (2) CGuard Embolic Prevention Stent $[60,61,70]$ (CGuard EPS, InspireMD), and (3) the Gore hybrid stent $[70,73,74]$ (Gore). Apart from the differences in the nitinol frame design (braided closed cell in RoadSaver/Casper, open cell in CGuard and the Gore hybrid stent), the three current double-layered carotid stent systems have other important design differences. These include the mesh material (braided nitinol in RoadSaver/ Casper, PET single-fibre knitted MicroNET in CGuard, and interwoven PTFE mesh in the Gore stent), the aperture size of the mesh pores $(500 \mu \mathrm{m}$ in the Gore stent, $375-500$ $\mu \mathrm{m}$ in RoadSaver/Casper, and only 150-180 $\mu \mathrm{m}$ in CGuard EPS), and the position of the mesh in relation to the nitinol frame (outside the frame for the CGuard EPS and Gore stent, and inside in the case of the RoadSaver/Casper) [60, $61,70,72]$. The RoadSaver/Casper stent delivery system is low-profile and is very flexible and, similar to the Wallstent, the stent is re-sheathable up to $50 \%$ of the released stent length, which may offer a procedural advantage to some operators. However, RoadSaver/Casper exhibits very significant elongation on implantation, reaching almost $30 \%$ of the nominal stent length [72].

Unsurprisingly, when minimising the protective mesh cell size the system delivery profile increases because the more protective micro-cells per stent frame area unit, the more mesh "fibres" are needed. The protective mesh material needs to be packed, along the stent frame, in the delivery system, so (as a general rule) the smaller the mesh cell size, the larger the delivery profile. The RoadSaver/Casper has the smallest delivery profile $(5 \mathrm{Fr})$ with the Gore stent in the middle (5 Fr or 6 Fr delivery profile dependent on the stent diameter). The CGuard EPS offers the largest open cell nitinol frame size among the current carotid stent systems $\left(21.66 \mathrm{~mm}^{2}\right.$ [5]) combined with the smallest mesh micro-cell size $\left(0.023-0.032 \mu \mathrm{m}^{2}\right)$, which corresponds to the pore size in filters used for distal protection at CAS, making the CGuard EPS (with 6 Fr outer diameter of the packed delivery system) the most "open" and, at the same time, the most "closed" cell carotid stent among the current designs [5]. CGuard EPS has a high radial force that is similar to the Precise stent (Cordis) and is higher than that of RoadSaver/Casper [61, 72]. On manufacturer-independent bench tests [61] CGuard EPS showed adaptation to vascular anatomies both in a step model and in a curved model that was superior to RoadSaver/Casper [61, 71].

Due to its construction, the RoadSaver/Casper does not have a tapered version. On the other hand, the 
CGuard EPS, with the SmartFit ${ }^{\top M}$ technology, has a property of self-tapering ("self-adaptation"), as demonstrated in bench tests [72] and confirmed on a systematic angiographic core lab evaluation in a series of over 100 CAS procedures [5]. Another CGuard EPS characteristic that may be important from the procedural standpoint is the lack of foreshortening or elongation both on bench tests [72] and in vivo [5, 72]; a feature that may offer an advantage particularly in anatomies that require precise stent placement [5]. It is thus important to realize that "mesh-covered" carotid stents form a very diverse family, and the fundamental design differences (including the mesh material and mesh position in relation to the stent frame) may translate into individual differences in embolic prevention efficacy on DW-MRI (that may or may not be clinically significant) as well as the device healing profile (including the risk of thrombosis and restenosis [70]), and that the implantation technique may also play a role to ensure proper device embedding into the vessel wall that may be possible with some but not other devices due to the mesh position and mesh material.

\section{Diffusion-weighted magnetic resonance imaging and mesh-covered stent systems embolic prevention efficacy}

The DW-MRI-depicted cerebral microembolisation is widely accepted as an index of the quality of cerebral protection during and after carotid revascularisation procedure $[43,55,60,75]$. The index (including prevalence of any new lesions, their size, and total number) is extremely sensitive because many small lesions totally resolve without any clinical sequelae [60]. Today, DW-MRI is an important tool in evaluating minimisation of intra-procedural embolisation with improved temporary neuroprotection systems $[35,38,40]$. The DW-MRI is similarly fundamental in evaluating the efficacy of different stent designs, in reducing peri-procedural embolisation, and preventing delayed cerebral embolism [67]. On the other hand, there is increasing evidence that carotid plaque-related cerebral embolisation may have a negative effect on cognitive function [76] (and intervention may be beneficial provided minimal intervention-related brain embolism [76-81]). Understanding is thus increasing today that the total burden of DW-MRI-depicted microembolisation in patients undergoing carotid revascularisation by CEA or CAS may be associated with transient cognitive decline [82]. In a proportion of patients, part of the deficit may persist months after the intervention $[82,83]$. Thus, any carotid intervention, to have a positive impact on brain function, needs to be associated with minimised cerebral embolism.

To date, the only published study with routine, per-protocol DW-MRI evaluation in all study patients rather than selected subjects appears to be the Carotid Embolic Protection Using MicroNET (CARENET) Multi- centric Trial [60]. CARENET demonstrated a low periprocedural embolism rate (37\%) with an extremely small lesion size $\left(0.039 \pm 0.08 \mathrm{~cm}^{3}\right)$ [60]. With the small lesion size with routine CGuard MicroNET-covered EPS use, there was resolution of the peri-procedural lesions by 30 days, and near-elimination of any post-procedural embolism with only one new minor $\left(0.116 \mathrm{~cm}^{3}\right)$ lesion in relation to the 48-h scan [60]. Comparison of the CARENET and PROFI data for patients treated under similar distal neuroprotection indicates a $\sim 2$-fold reduction in the prevalence of any DW-MRI lesion(s) and over 10-fold reduction in mean lesion volume when CGuard EPS rather than a conventional carotid stent is used $[60,75]$. Recently, the DW-MRI findings from the CARENET trial have been replicated by the Torino Vascular Radiology Group [84], who reported the new DW-MRI lesion prevalence of $36.5 \%$ and an average 0.55 lesions per patient with median lesion volume of $0.040 \mathrm{~cm}^{3}$ (IQR: $0.025-0.050 \mathrm{~cm}^{3}$ ) using the CGuard EPS [84]. For the RoadSaver/Casper stent, the Torino Group found DW-MRI lesion prevalence of $37.5 \%$, with an average 1.3 lesions per patient, and median lesion volume of $0.076 \mathrm{ml}$ (IQR: $0.064-0.138 \mathrm{~cm}^{3}$ ) [84]. Most recently, the IRON-GUARD study reported new post-procedural DW-MRI lesions in only 12 (19.6\%) out of 61 patients subjected to DW-MRI imaging in CAS using CGuard EPS and mainly distal EPD [63].

\section{Mesh-covered carotid stents: accumulating clinical evidence}

The article by Machnik et al. [62] in the current issue of the Journal provides an important contribution to the growing clinical evidence for a low neurological complication rate with the novel mesh-covered [10,69] carotid stents. The single stroke in the series of mainly symptomatic $(51.2 \%)$ patients, treated by these operators [62] using the Roadsaver/Casper stent [71, 72] under predominantly proximal (65.8\%) embolic protection, was a minor stroke that occurred after the common carotid artery cannulation with guiding catheter, i.e. prior to stent insertion and implantation [62].

With the patient series by Machnik et al. [62], the present body of clinical evidence has grown, considering studies that included at last 30 patients, to 551 CAS patients treated with mesh-covered carotid stents. This involved 190 subjects treated with Roadsaver/Casper [6, $7,62]$ and 361 CAS patients treated using CGuard EPS $[5,60,61,63]$. These studies enrolled up to $\sim 50 \%$ of symptomatic patients $[5,62]$ and were inclusive, in the all-comer series [5], of patients with acute symptoms of cerebral ischaemia (TIA or acute stroke in transition [5]). Inclusive of the periprocedural neurologic events that occurred prior to stent placement, 30-day follow-up in those 551 CAS patients (558 CAS procedures) showed only 6 peri-procedural strokes (1.08\%) [5-7, 60-63] and only $1(0.18 \%)$ post-procedural stroke, which occurred in a pa- 
tient with concomitant atrial fibrillation in the absence of adequate anticoagulation [6]. Thus the overall 30-day event rate was only $1.26 \%$ [5-7, 60-63]. These results are remarkable, particularly as reassuring 12-month reports from the CGuard EPS studies are arriving in the public domain in 2017 [85, 86]. At this point, it may be premature, however, to call the conventional carotid stents a historical footnote because still more evidence and longer follow-ups are required [87].

\section{Mesh-covered carotid stents: what further evidence do we need?}

At the present stage, the mesh-covered carotid stent field, with data from over 550 patients and an overall procedural event rate of $\sim 1 \%$ with near-elimination of post-procedural events during stent healing [5-7, 60-63], is ready for a formal statistical overview of the results using individual patient data from the published studies. While this important clinical evidence (supplemented by routine DW-MRI data in all consecutive patients in one trial [60] and DW-MRI imaging in some patients in other studies $[7,61,63,84])$ is already transforming the carotid revascularisation field, it is not sufficient to firmly establish the role of the novel technology [87].

Randomised studies would be particularly welcome because these are the ones that can provide 'ultimate' level-1 evidence [87], despite the fact that the lesson from CREST $[21,24]$ shows that acceptance of the study message may depend on what the message is [22]. As we contemplate future trials, it is important to understand that the data from the randomised studies either in terms of clinical endpoints or intervention-associated DW-MRI lesion incidence, intensity, total lesion number, and size, will no doubt continue to play an important role as a clinically-relevant surrogate endpoint $[10,75,81]$. Outcomes will critically depend on what patients (symptomatic vs. asymptomatic, proportion of symptomatics) and lesions (not critical, stable, but angiographically significant "to meet" the revascularisation indications vs. increased-embolism-risk, ulcerated, or thrombus-containing/critical stenoses [10]) are enrolled and undergo randomisation. To demonstrate unequivocally the benefit of the new technology, the randomised studies "should" include the high (embolisation)-risk subjects because this is the group that expected to show the most profound difference after randomisation. While including high (embolisation)-risk subjects is right (and required) from the point of study methodology, it may be questionable from the ethical standpoint [10]. This is particularly relevant because the data already available in the public domain indicate $\sim 50 \%$ reduction in the incidence of cerebral embolisation and $\sim 10$-fold reduction in mean lesion volume [60] when comparing the MicroNET-covered stent to a conventional carotid stent design used under the same type of temporary neuroprotection [75]. This concern may be somewhat mitigated by enrolling only those patients who are high risk for CEA, since many patients at high risk for CEA can be safely treated with CAS. Randomised trials remove the treatment selection bias but, as demonstrated by several recent examples in cardiovascular medicine, they are greatly sensitive to patient selection bias $[88,89]$. Patient selection bias may lead paradoxically to removing from the study ("patients not subjected to randomisation") those to whom the evaluated intervention is predominantly addressed $[88,89]$. For instance, in the recent TASTE study one of the main reasons to exclude subjects with thrombus-containing coronary lesions from the cohort undergoing randomisation in a major clinical trial of coronary thrombus extraction in acute myocardial infarction [88] was thrombus burden requiring, in the operator's judgment, thrombus aspiration/removal. This was not indicated in the study inclusion/exclusion criteria but it was listed as one of the principal reasons why patients meeting the inclusion criteria did not undergo randomisation [88]. While this was no doubt right ethically, it was clearly wrong methodologically, resulting in lowrisk subject enrolment and (unsurprisingly) an apparent "lack of benefit" of thrombus aspiration [89], particularly because the study was focused on a low-incidence endpoint of mortality [88]. While this provided zero evidence for the futility of routine consideration of thrombus aspiration in acute myocardial infarction, it led (surprisingly) to a "downgrade" in the role of this intervention in the guidelines from Class Ila (weight of evidence in favour of usefulness/efficacy - intervention should be considered) [90] to Class IIb (usefulness/efficacy is less well established - intervention may be considered) [91] because of the new "randomised study evidence". The mesh-covered stent technology requires awareness to avoid a similar trap because, for many CAS operators, it might be already ethically unacceptable today to randomise high-embolism-risk patients (such as symptomatic or with thrombotic lesions) to an open-cell carotid stent vs. a MicroNET-covered stent $[5,10]$, whereas the randomised study outcome may be critically dependent on inclusion of such high (embolisation)-risk subjects, particularly if focused on clinical end-points such as peri-procedural stroke and stroke during device healing (30-day stroke).

On routine DW-MRI cerebral imaging, CGuard EPS system use was associated with $>50 \%$ reduction in the incidence of peri-procedural DW-MRI lesions and >10fold reduction in average lesion volume $[60,74]$. With the DW-MRI randomised data desired (but due to cost and logistics unlikely to be performed on a very large scale), any future comparison should correct for the proximal vs. distal temporary protection type (which plays a role in minimising the intra-procedural but not post-procedural cerebral embolisation [31, 35, 36, 38, 39, 40, 43]) and the type of lesion(s)/symptomatic status.

Because evidence from clinical studies should be applicable to routine clinical practice, the role of all-comer 
study protocols is increasingly understood in cardiovascular medicine $[5,92]$ to minimise the proportion of trialunrepresented patients $[88,89]$ to whom the tested intervention is designed to apply. Routine access to tools that enable achievement of CAS 30-day complication rate $\leq 1 \%$ in truly all-comer populations comprised of a majority of symptomatic patients and including only increasedstroke-risk asymptomatic patients [5], transforms the carotid revascularisation field [10, 93]. All-comer carotid referral tracking in a tertiary cardiovascular centre suggested recently that $>90 \%$ (not all) contemporary carotid stenosis patients with NeuroVascular Team-determined indication to revascularisation (cohort including > 50\% symptomatic patients, whereas the asymptomatic patients required at least one increased stroke-risk criterion) can be safely and effectively treated using the endovascular route and a mesh-covered carotid embolic prevention stent system [5]. On the other hand, there are patient subsets where, for a vascular surgeon, CAS is clearly preferred over CEA due to anatomical or co-morbidity criteria [6]. There is (and will remain!) a group of revascularisation-indicated carotid stenosis patients who are natural candidates for surgery, a group - on the other side of the spectrum - with preferred endovascular management [6], and a very large group in the middle that can be managed with either method [5].

The multicentric multi-specialty CARENET trial demonstrated evidence for near-elimination of new lesion occurrence during the MicroNet-covered CGuard EPS healing period [60]. Thus, systematic peri-procedural and 30-day DW-MRI data on the two other mesh-covered stent systems (RoadSaver/Casper and the Gore hybrid stent) in cohorts of unselected patients are awaited to confirm the anti-embolic efficacy of these stents and to see whether there is any signal of a potential effect of design differences on embolic prevention efficacy [70, 84].

It remains to be determined whether the stent design and mechanical properties differences are important in terms of clinical safety and efficacy including, for instance, the risk of stent thrombosis or restenosis [94]. Moreover, the stent implantation technique (including post-dilatation to maximise embedding) may play a role. The double-layer stent design is naturally associated with permanent implantation of more material than in the case of a single-layered traditional carotid stent. This might, at least with some designs, affect stent healing and might, at least in theory, be related with a higherthan-in-a-single-layer stent propensity to stent thrombosis and restenosis. The thrombosis risk may be further influenced by the stent design through the combined thickness of the two layers (that significantly differs with different "mesh-covered" stent designs) and with the implantation technique, both resulting in a higher or lower malapposition rate. The combined thickness of the double-layer may influence the device healing duration. Similarly, the use of braided mesh design as opposed to non-metallic materials for enhanced plaque coverage with increased metal surface area exposed to platelets in the blood stream may pose unclear risks for stent thrombosis or delayed re-stenosis. In that context, optimal implantation technique (including the post-dilatation/ optimisation strategy [5]) and duration of clopidogrel use in addition to life-long aspirin, or the place of other drugs, such as ticagrelor, with the different stent designs remains undetermined [10].

During CAS, cerebral embolisation can (and does, albeit not in all patients) occur at the stage of aortic and target artery cannulation, guide catheter or sheath placement, lesion wiring, filter crossing (for distal-protected CAS), predilatation (if performed), stent placement across the lesion, and stent post-dilatation as well as post implantation through plaque protrusion [39, 51]. Because, with the dual-layer stent design, the mesh offers protection against plaque material cerebral embolisation only starting with the stent post-dilatation phase, it is certainly premature to call off the temporary brain protection devices in CAS using dual-layer stent systems [6]. This is particularly relevant in the absence of any criteria for temporary neuroprotection device use (vs. non-use) [6] and because the embolic material (unsurprisingly in the context of emboli-generating CAS stages prior to establishing the mesh protection) continues to be present, albeit at an apparently lower rate [5, 37], in the intra-procedural (temporary) protection systems employed in CAS employing exclusively the novel stent generation [5]. Therefore, the concept of "no-need-for-neuroprotection device" with a dual-layered stent use [6], although definitely attractive to some operators, requires large-scale, properly designed investigation with routine DW-MRI imaging before any potential application of the strategy in some patient/lesion subsets, such as the treatment of conventional carotid stent in-stent restenosis (note the need to differentiate between true restenosis and neo-atherosclerosis) or primary stenting of a fibrotic non-critical lesion (in the case of a clinical indication for revascularisation). It is also important to realise that the supposedly "benign" subset of conventional carotid stent restenotic lesions include instances of severe neo-atherosclerosis [66] (continued intraluminal growth of the atherosclerotic plaque between the struts of the conventional, uncovered, stent struts - up to the point of intervention-requiring lumen compromise, clinical symptoms, or signs of embolisation on brain imaging) with a very high risk of intraprocedural cerebral embolism [66].

Another important issue in the emerging field is to establish the role of direct carotid artery angioplasty and stenting (trans-carotid arterial revascularisation - TCAR, using surgical vascular access and endovascular dynamic flow reversal, which is highly effective in minimising intra-procedural cerebral embolisation [35, 36, 38, 42, 65]) in combination with the novel dual-layer carotid stent technology for sustained embolic prevention. Initial ex- 
perience of TCAR CAS combined with MicroNET-covered CGuard EPS use in a high cerebral risk cohort (73\% symptomatic lesions, contralateral internal carotid artery occlusion in $27 \%$, ipsilateral ischaemic cerebral lesions on baseline DW-MRI in 100\% of subjects), evidence by routine peri-procedural DW-MRI cerebral imaging combined with post-procedural DW-MRI extended to 90 days after (64\% of procedures under local anaesthesia, dynamic flow reversal time from $6 \mathrm{~min} 20 \mathrm{~s}$ to $11 \mathrm{~min} 30 \mathrm{~s}$, median 7 min 50 s), showed - with $100 \%$ clinical and neurological follow-up and 100\% DW-MRI cerebral imaging at 90 days - (1) no neurological or other complications, (2) near-elimination of peri-procedural cerebral embolism (only $0.36 \mathrm{DW}-\mathrm{MRI}$ lesion per patient), and (3) total elimination of post-procedural cerebral embolism throughout the 90-day [66] period that exceeds stent healing duration [70]. This is consistent with the concept that the two technologies (TCAR dynamic flow reversal plus CGuard MicroNET-covered EPS), when combined, act in a complementary fashion (efficient capture and removal of intra-procedural debris with TCAR plus minimisation of intra-procedural embolism and sustained elimination of post-procedural embolism with CGuard EPS) and may be extremely efficient together [66], leading to a significant impact on evolution of the carotid revascularisation field.

Last but not least, an urgent, multi-specialty, and large-scale endeavour, involving neurologists, neurointerventionalists, vascular surgeons, radiologists, cardiologists, and angiologists is greatly needed to close the embarrassing gap in prospectively validated evidence to determine the optimal management paths for subjects with asymptomatic carotid stenosis including effective risk-stratification tools [9-12, 95, 96].

\section{Conclusions}

Atherosclerotic plaque protrusion through conventional carotid stent struts [8, 56-59] has been identified as a leading mechanism of post-procedural cerebral embolisation [55] and post-procedural adverse neurological events seen with the prior generation of carotid stents $[47,48,50,54]$. Vascular surgery key opinion leaders have repeatedly indicated that the safety of CAS needs to be improved before CAS can be routinely applied by surgeons as an alternative to CEA $[68,69]$, and that the CAS role in carotid revascularisation would be fully established only with an increased use of proximal neuroprotection systems and the development of novel "ultra-closed" cell stent systems [68].

Evidence is accumulating today for an adequate answer from the endovascular community (with a highly significant impact from endovascular-minded surgical colleagues and centres $[5,6,63])$ to the surgical postulate of improved carotid stent designs $[68,69]$ (to minimise intra-procedural embolism and prevent post-procedural embolisation [60]) and wider acceptance of more effective embolic protection such as proximal protection devices for higher (embolic)-risk patients in particular [6, 34, 36, 66]. The spring of improvements in carotid stent design $[60,70$, 72] and increased understanding of the role of optimised temporary brain protection at CAS [31-34, 36, 39, 40, 42, 97], taken hand-in-hand with improvements in medical therapy [13], is already turning into the summer of routine clinical applications to prevent carotid atherosclerosis-related strokes. Emerging clinical experience indicates that the long-awaited $[68,69]$ technological advancement of double-layer "mesh" stents has widely re-opened the endovascular route viability to the majority of patients with carotid stenosis and NeuroVascular Team-recommended $[5,10]$ indication to carotid revascularisation.

Large-scale level 1 evidence for the efficacy of dual-layer carotid stent systems including clinical endpoints would be highly desirable, similar to the need to prospectively establish the role of intervention in asymptomatic carotid stenosis [9-11, 95] and tools to identify increased-stroke-risk subjects to target the intervention to those most likely to benefit from it $[12,81,96]$. Experience from prior large-scale work, however, shows that such studies may be bound to practically suffer from the problems of effective recruitment volume/patient cross over and funding [24, 98, 99]. Selection bias-free randomisation could be considered either unethical (in the case of high-risk lesion randomisation in a study employing prior generation single-layer stent(s) now DW-MRI evidenced to be associated with cerebral embolisation significantly exceeding, in both incidence and volume, that of a mesh-covered stent system $[5,60])$, or the study would be a priori likely to be inconclusive (in case of lowrisk patient lesion randomisation). Cerebral DW-MRI imaging is going to continue $[35,38,40,43,55,60,67]$ to provide a sensitive and clinically-relevant [76, 79, 80, 83] surrogate in the evaluation of novel carotid revascularisation technologies and treatment strategies.

Because knowledge from CREST has led to the evolution of both CAS and CEA, any continuation of the discussion based on the CREST composite endpoint individuals ("would you rather have a (minor) stroke (with CAS)?" or "would you rather have a myocardial infarction (MI) (with (EA)?") [22] is becoming obsolete today because such a discussion is largely irrelevant to contemporary knowledge and practice. The Gordian knot of the relative importance of the composite endpoint individuals in CREST $[22,23]$ is getting cut by the progress in endovascular medicine and surgery. There are, and will remain, patient and lesion subsets that will be better off with endovascular management and, on the other side of the spectrum, patient and lesion subsets that should be treated with surgery as a first-line strategy. But because the common area is large, patients and families should be given access to full and unbiased information to make conscious choices [100], taking into account the technological developments and new medical knowledge arising from 
contemporary (rather than historic) studies. Routine access to tools and techniques that enable achievement of CAS complication rates of $\sim 1 \%$ at 30 days [5-7, 10, 60-63, 93] are transforming the endovascular carotid revascularisation field. In the (not remote) past, when different revascularisation techniques preferentially (almost) "belonged" to different specialties, a question of "how? (should carotid revascularisation be performed)" was effectively the question of "who? (should perform carotid revascularisation)". This may have led to a bias in how the particular revascularisation strategy/technology was being presented to referring communities (such as neurology or non-interventional vascular medicine), positioned in the guidelines and, effectively, reimbursed (including implementation of specialty-selective reimbursement pathways in some healthcare systems).

Today, with a growing proportion of endovascular-minded surgeons who routinely apply endovascular techniques in their every-day practice to treat common vascular pathologies, and with the advanced stent technologies [5-7, 60-63] that enable sustained embolic prevention $[60,66]$ and improved peri-procedural cerebral protection strategies [35-38, 43-45, 65, 66, 101], the surgical community considers CAS a viable prospective treatment modality in carotid revascularisation [93, 97]. Rather than acutely treated (which is generally accepted despite the fact that, for many patients, it comes unfortunately too late to avoid clinically-relevant brain damage [3-5]), carotid stenosis-related strokes should be safely and effectively prevented [10-12]. The spring of technological progress is now turning into the summer of routine clinical applications. "How?", "using what technology?", and "with what experience and expertise?" should, at last, matter more than "who?" should be performing carotid artery revascularisation in acute stroke management as well as in primary and secondary stroke prevention.

\section{Conflict of interest}

PM: Advisory Board/Consulting/Speaker Bureau: Abbott, InspireMD, Medtronic; Research Support: Abbott, Jagiellonian University School of Medicine, Ministry of Science - Poland, 'For The Heart' Foundation in Krakow, Polish Cardiac Society/ADAMED Grant - Basic Research in Carotid Disease, Polish Cardiac Society/Servier Grant - Clinical Research in Carotid Disease; PI/Steering Committees: CIRCULATE - STRATEGMED2, CARENET, PARADIGM, PARADIGM-EXTEND; Financial Interest: None. LNH: Consulting: Boston Scientific, Cordis, Abbott, Medtronic; Research Support: Toshiba; Board, Trustee, or Officer Position: Claret Medical, Inc; Honorarium: Memorial Healthcare System, Complete Conf. Management; Financial Interests: Boston Scientific, Claret Medical Inc, Augmenix, Endomation, Silk Road, Ostial, Apama, StimSox, Photolitec, ValenTx, Axtria, Ocular, The Stroke Project Inc. AHS: Consulting: Amnis Therapeutics Ltd, Cerebrotech Medical
Systems Inc, CereVasc LLC, Claret Medical Inc, Codman, Corindus Inc, GuidePoint Global Consulting, Medtronic (Formerly Covidien), MicroVention, Neuravi, Penumbra, Pulsar Vascular, Rapid Medical, Rebound Therapeutics Corporation, Silk Road Medical, Stryker, The Stroke Project Inc, Three Rivers Medical Inc, W.L. Gore \& Associates; Board Member: Intersocietal Accreditation Commission; PI/Steering Committees: Codman \& Shurtleff, LARGE Trial, Covidien (Now Medtronic), SWIFT PRIME and SWIFT DIRECT Trials; MicroVention, FRED Trial, CONFIDENCE Study, MUSC, POSITIVE Trial; Penumbra, 3D Separator Trial, COMPASS Trial, INVEST Trial; Financial interests: Buffalo Technology Partners Inc, Cardinal, International Medical Distribution Partners, Medina Medical Systems, Neuro Technology Investors, StimMed, Valor Medical.

\section{References}

1. Mozaffarian D, Benjamin EJ, Go AS, et al. Heart Disease and Stroke Statistics - 2016 Update: a Report from the American Heart Association. Circulation 2016; 133: e38-60.

2. Rangel-Castilla L, Rajah GB, Shakir HJ, et al. Endovascular prevention and treatment of stroke related to extracranial carotid artery disease. J Cardiovasc Surg 2017; 58: 35-48.

3. Mpotsaris A, Kabbasch C, Borggrefe J, et al. Stenting of the cervical internal carotid artery in acute stroke management: the Karolinska experience. Interv Neuroradiol 2017; 23: 159-65.

4. Kappelhof M, Marquering HA, Berkhemer OA, Majoie CB. Intra-arterial treatment of patients with acute ischemic stroke and internal carotid artery occlusion: a literature review. J Neurointerv Surg 2015; 7: 8-15.

5. Musialek P, Mazurek A, Trystula M, et al. Novel PARADIGM in carotid revascularisation: prospective evaluation of All-comer peRcutaneous cArotiD revascularisation in symptomatic and Increased-risk asymptomatic carotid artery stenosis using CGuard $^{\mathrm{TM}}$ MicroNet-covered embolic prevention stent system. Eurolntervention 2016; 12: e658-70.

6. Bosiers M, Deloose K, Torsello G, et al. The CLEAR-ROAD study: evaluation of a new dual layer micromesh stent system for the carotid artery. Eurolntervention 2016; 12: e671-6.

7. Nerla R, Castriota F, Micari A, et al. Carotid artery stenting with a new-generation double-mesh stent in three high-volume Italian centres: clinical results of a multidisciplinary approach. Eurolntervention 2016; 12: e677-83.

8. Kotsugi M, Takayama K, Myouchin K, et al. Carotid artery stenting: investigation of plaque protrusion incidence and prognosis. JACC Cardiovasc Interv 2017; 10: 824-31.

9. Conrad MF, Boulom V, Mukhopadhyay S, et al. Progression of asymptomatic carotid stenosis despite optimal medical therapy. J Vasc Surg 2013; 58: 128-35.

10. Musialek P, Hopf-Jensen S. Carotid artery revascularization for stroke prevention: a new era. J Endovasc Ther 2017; 24: 138-48.

11. Sabeti S, Schlager O, Exner M, et al. Progression of carotid stenosis detected by duplex ultrasonography predicts adverse outcomes in cardiovascular high-risk patients. Stroke 2007; 38: 2887-94.

12. Paraskevas KI, Nicolaides AN, Veith FJ. Carotid endarterectomy may be required in addition to best medical treatment for some patient subgroups with asymptomatic carotid stenosis. Vascular 2015; 23: 62-4. 
13. Paraskevas KI, Mikhailidis DP, Veith FJ, Spence JD. Definition of best medical treatment in asymptomatic and symptomatic carotid artery stenosis. Angiology 2016; 67: 411-9.

14. Fisher M, Paganini-Hill A, Martin A, et al. Carotid plaque pathology: thrombosis, ulceration, and stroke pathogenesis. Stroke 2005; 36: 253-7.

15. Virmani R, Ladich ER, Burke AP, Kolodgie FD. Histopathology of carotid atherosclerotic disease. Neurosurgery 2006; 59 (Suppl 3): S219-27.

16. Kernan WN, Ovbiagele B, Black HR, et al. Guidelines for the prevention of stroke in patients with stroke and transient ischemic attack: a guideline for healthcare professionals from the American Heart Association/American Stroke Association. Stroke 2014; 45: 2160-236.

17. Widimsky P, Kolh P, Agewall, et al. 2017 European Society of Cardiology (ESC) Guidelines on the diagnosis and Treatment of Peripheral Arterial Disease - in collaboration with the European Society of Vascular Surgery (ESVS) and European Stroke Organization (ESO). Eur Heart J 2017 (in press).

18. Eller JL, Snyder KV, Siddiqui AH, et al. Endovascular treatment of carotid stenosis. Neurosurg Clin N Am 2014; 25: 565-82.

19. Papanagiotou $P$, Roth $C$, Walter $S$, et al. Carotid artery stenting in acute stroke. J Am Coll Cardiol 2011; 58: 2363-9.

20. Wach MM, Dumont TM, Mokin M, et al. Early carotid angioplasty and stenting may offer non-inferior treatment for symptomatic cases of carotid artery stenosis. J Neurointerv Surg 2014; 6: 276-80.

21. Brott TG, Howard G, Roubin GS, et al. Long-term results of stenting versus endarterectomy for carotid-artery stenosis. N Engl J Med 2016; 374: 1021-31.

22. Mousa A, Broce M. Carotid endarterectomy before and after CREST. J Endovasc Ther 2016; 23: 536-7.

23. Aksoy O, Kapadia SR, Bajzer C, et al. Carotid stenting vs surgery: parsing the risk of stroke and MI. Cleve Clin J Med 2010; 77: 892-902.

24. Brott TG, Hobson RW $2^{\text {nd }}$, Howard G, et al. Stenting versus endarterectomy for treatment of carotid-artery stenosis. N Engl J Med 2010; 363: 11-23.

25. Sardar P, Chatterjee S, Aronow HD, et al. Carotid artery stenting versus endarterectomy for stroke prevention: a meta-analysis of clinical trials. J Am Coll Cardiol 2017; 69: 2266-75.

26. Hill MD, Brooks W, Mackey A, et al. Stroke after carotid stenting and endarterectomy in the Carotid Revascularization Endarterectomy versus Stenting Trial (CREST). Circulation 2012; 126: 3054-61.

27. Blackshear JL, Cutlip DE, Roubin GS, et al. Myocardial infarction after carotid stenting and endarterectomy: results from the carotid revascularization endarterectomy versus stenting trial. Circulation 2011; 123: 2571-8.

28. Stilp E, Baird C, Gray WA, et al. An evidence-based review of the impact of periprocedural myocardial infarction in carotid revascularization. Catheter Cardiovasc Interv 2013; 82: 709-14.

29. Paraskevas KI, Kalmykov EL, Naylor AR. Stroke/death rates following carotid artery stenting and carotid endarterectomy in contemporary administrative dataset registries: a systematic review. Eur J Vasc Endovasc Surg 2016; 51: 3-12.

30. Chaturvedi S, Madhavan R, Santhakumar S, et al. Higher risk factor burden and worse outcomes in urban carotid endarterectomy patients. Stroke 2008; 39: 2966-8.
31. Nikas D, Reith W, Schmidt A, et al. Prospective, multicenter European study of the GORE flow reversal system for providing neuroprotection during carotid artery stenting. Catheter Cardiovasc Interv 2012; 80: 1060-8.

32. Ansel GM, Hopkins LN, Jaff MR, et al. Safety and effectiveness of the INVATEC MO.MA proximal cerebral protection device during carotid artery stenting: results from the ARMOUR pivotal trial. Catheter Cardiovasc Interv 2010; 76: 1-8.

33. Clair DG, Hopkins LN, Mehta M, et al. Neuroprotection during carotid artery stenting using the GORE flow reversal system: 30-day outcomes in the EMPiRE Clinical Study. Catheter Cardiovasc Interv 2011; 77: 420-9.

34. Mokin M, Dumont TM, Chi JM, et al. Proximal versus distal protection during carotid artery stenting: analysis of the two treatment approaches and associated clinical outcomes. World Neurosurg 2014; 81: 543-8.

35. Pinter L, Ribo M, Loh C, et al. Safety and feasibility of a novel transcervical access neuroprotection system for carotid artery stenting in the PROOF Study. J Vasc Surg 2011; 54: 1317-23.

36. Kwolek CJ, Jaff MR, Leal J, et al. Results of the ROADSTER multicenter trial of transcarotid stenting with dynamic flow reversal. J Vasc Surg 2015; 62: 1227-34.

37. Pieniazek P, Musialek P, Kablak-Ziembicka A, et al. Carotid artery stenting with patient- and lesion-tailored selection of the neuroprotection system and stent type: early and 5-year results from a prospective academic registry of 535 consecutive procedures (TARGET-CAS). J Endovasc Ther 2008; 15: 249-62.

38. Leal I, Orgaz A, Flores Á, et al. A diffusion-weighted magnetic resonance imaging-based study of transcervical carotid stenting with flow reversal versus transfemoral filter protection. J Vasc Surg 2012; 56: 1585-90.

39. Montorsi P, Caputi L, Galli S, et al. Microembolization during carotid artery stenting in patients with high-risk, lipid-rich plaque. A randomized trial of proximal versus distal cerebral protection. J Am Coll Cardiol 2011; 58: 1656-63.

40. Stabile E, Sannino A, Schiattarella GG, et al. Cerebral embolic lesions detected with diffusion-weighted magnetic resonance imaging following carotid artery stenting: a meta-analysis of 8 studies comparing filter cerebral protection and proximal balloon occlusion. JACC Cardiovasc Interv 2014; 7: 1177-83.

41. Pieniążek P, Tekieli L, Musiałek P, et al. Carotid artery stenting according to the tailored-CAS algorithm is associated with a low complication rate at 30 days: data from the TARGET-CAS study. Kardiol Pol 2012; 70: 378-86.

42. Paraskevas KI, Veith FJ, Parodi JC. Transcervical carotid artery senting (CAS) with flow reversal: a promising technique for the reduction of strokes associated with CAS. J Endovasc Ther 2016; 23: 255-7.

43. Grunwald IQ, Reith W, Kühn AL, et al. Proximal protection with the Gore PAES can reduce DWI lesion size in high-grade stenosis during carotid stenting. Eurolntervention 2014; 10: 271-6.

44. Cremonesi A, Gieowarsingh S, Spagnolo B, et al. Safety, efficacy and long-term durability of endovascular therapy for carotid artery disease: the tailored-Carotid Artery Stenting Experience of a single high-volume centre (tailored-CASE Registry). EuroIntervention 2009; 5: 589-98.

45. Pieniazek P, Musialek P, Dzierwa K, et al. Flow reversal for proximal neuroprotection during endovascular management of critical symptomatic carotid artery stenosis coexisting with ipsilateral external carotid artery occlusion. J Endovasc Ther 2009; 16: 744-51. 
46. Bosiers M, de Donato G, Deloose K, et al. Does free cell area influence the outcome in carotid artery stenting? Eur J Vasc Endovasc Surg 2007; 33: 135-41.

47. Tietke M, Jansen O. Cerebral protection vs. no cerebral protection: timing of stroke with CAS. J Cardiovasc Surg 2009; 50: 751-60.

48. Doig D, Turner EL, Dobson J, et al. Predictors of stroke, myocardial infarction or death within 30 days of carotid artery stenting: results from the International Carotid Stenting Study. Eur J Vasc Endovasc Surg 2016; 51: 327-34.

49. Tatli E, Tokatli A, Vatan MB, et al. Comparison of closed-cell and hybrid-cell stent designs in carotid artery stenting: clinical and procedural outcomes. Adv Interv Cardiol 2017; 13: 135-41.

50. Stabile E, Giugliano G, Cremonesi A, et al. Impact on outcome of different types of carotid stent: results from the European Registry of Carotid Artery Stenting. Eurolntervention 2016; 12: e265-70.

51. Al-Mubarak N, Roubin GS, Vitek JJ, et al. Effect of the distal-balloon protection system on microembolization during carotid stenting. Circulation 2001; 104: 1999-2002.

52. Macdonald S, Evans DH, Griffiths PD, et al. Filter-protected versus unprotected carotid artery stenting: a randomised trial. Cerebrovasc Dis 2010; 29: 282-9.

53. Tulip $\mathrm{HH}$, Rosero EB, Higuera AJ, et al. Cerebral embolization in asymptomatic versus symptomatic patients after carotid stenting. J Vasc Surg 2012; 56: 1579-84.

54. Fairman R, Gray WA, Scicli AP, et al. The CAPTURE registry: analysis of strokes resulting from carotid artery stenting in the post approval setting: timing, location, severity, and type. Ann Surg 2007; 246: 551-6.

55. Schofer J, Arendt M, Tübler T, et al. Late cerebral embolization after emboli-protected carotid artery stenting assessed by sequential diffusion-weighted magnetic resonance imaging. JACC Cardiovasc Interv 2008; 1: 571-7.

56. Reimers B, Nikas D, Stabile E, et al. Preliminary experience with optical coherence tomography imaging to evaluate carotid artery stents: safety, feasibility, and techniques. Eurolntervention 2011; 7: 98-105.

57. De Donato G, Setacci F, Sirignano P, et al. Optical coherence tomography after carotid stenting: rate of stent malapposition, plaque prolapse and fibrous cap rupture according to stent design. Eur J Vasc Endovasc Surg 2013; 45: 579-87.

58. Shinozaki N, Ogata N, Ikari Y. Plaque protrusion detected by intravascular ultrasound during carotid artery stenting. J Stroke Cerebrovasc Dis 2014; 23: 2622-5.

59. Liu R, Jiang Y, Xiong Y, et al. An optical coherence tomography assessment of stent strut apposition based on the presence of lipid-rich plaque in the carotid artery. J Endovasc Ther 2015; 22: 942-9.

60. Schofer J, Musiałek P, Bijuklic K, et al. A prospective, multicenter study of a novel mesh-covered carotid stent: the CGuard CARENET Trial (Carotid Embolic Protection Using MicroNET). JACC Cardiovasc Interv 2015; 8: 1229-34.

61. Wissgott C, Schmidt W, Brandt-Wunderlich C, et al. Clinical results and mechanical properties of the CGuard double-layered embolic protection stent. J Endovasc Ther 2017; 24: 130-7.

62. Machnik R, Paluszek P, Tekieli L, et al. Mesh-covered (Roadsaver) stent as a new treatment modality for symptomatic or high-risk carotid stenosis. Adv Interv Cardiol 2017; 13: 130-4.

63. Speziale F, Capoccia L, Sirignano P, et al. 30-day results from prospective multi-specialty evaluation of carotid artery stenting using the CGuard micronet-covered embolic prevention stent system in real world multicenter clinical practice: the IRON-GUARD study. Eurolntervention 2017 May 9 [Epub ahead of print]

64. Ziaja K, Kuczmik W. Carotid artery stenting: In whom? When? How? And by whom? - The vascular surgeon point of view [Polish]. Medycyna Praktyczna - Kardiologia (kardiologia.mp.pl) 2009; 3: 5-12.

65. Plessers M, Van Herzeele I, Hemelsoet D, et al. Transcervical carotid stenting with dynamic flow reversal demonstrates embolization rates comparable to carotid endarterectomy. J Endovasc Ther 2016; 23: 249-54.

66. Musialek P, Trystula M. Impact Of TCAR And Micro-Mesh Stent Usage With The C-Guard Stent (Inspire MD) On CAS: Do They Decrease DW MRI Cerebral Embolic Lesions and Which Helps Most? Invited Lecture at VEITH Congress (Vascular Endovascular Issues Techniques Horizons, New York, NY, USA, presented on November 18, 2016, available at http://www.veithsymposium.org/abstracts/2016/vei/737.pdf; accessed 12 June 2017).

67. Park KY, Kim DI, Kim BM, et al. Incidence of embolism associated with carotid artery stenting: open-cell versus closed-cell stents. J Neurosurg 2013; 119: 642-7.

68. Paraskevas KI, Mikhailidis DP, Veith FJ. Mechanisms to explain the poor results of carotid artery stenting (CAS) in symptomatic patients to date and options to improve CAS outcomes. J Vasc Surg 2010; 52: 1367-75.

69. Mas JL, Trinquart L, Leys D, et al. Endarterectomy Versus Angioplasty in Patients with Symptomatic Severe Carotid Stenosis (EVA-3S) trial: results up to 4 years from a randomised, multicentre trial. Lancet Neurol 2008; 7: 885-92.

70. Musialek P. Mesh-Covered Stents for Carotid Intervention: Rationale, Device Designs, Imaging, and Data to Date Invited. Lecture at Transcatheter Cardiovascular Therapeutics (TCT 2015), Carotid and Stroke Intervention: State of the Art Session, October 14, 2015. Available at http://www.tctmd.com/show.aspx?id=132421 (accessed 12 June 2017).

71. Hopf-Jensen S, Marques L, Preiß M, Müller-Hülsbeck S. Initial clinical experience with the micromesh Roadsaver carotid artery stent for the treatment of patients with symptomatic carotid artery disease. J Endovasc Ther 2015; 22: 220-5.

72. Wissgott C, Schmidt W, Brandt-Wunderlich C, et al. Preliminary clinical results and mechanical behavior of a new double-layer carotid stent. J Endovasc Ther 2015; 22: 634-9.

73. Schönholz C, Yamada R, Montgomery W, et al. First-in-man implantation of a new hybrid carotid stent to prevent periprocedural neurological events during carotid artery stenting. J Endovasc Ther 2014; 21: 601-4.

74. Yamada R, Guimaraes M, Adams J, Schönholz C. New technologies for CAS that might overcome the burden of microembolization. J Cardiovasc Surg 2015; 56: 859-65.

75. Bijuklic K, Wandler A, Hazizi F, Schofer J. The PROFI study (Prevention of Cerebral Embolization by Proximal Balloon Occlusion Compared to Filter Protection During Carotid Artery Stenting): a prospective randomized trial. J Am Coll Cardiol 2012; 59: 1383-9.

76. Siddiqui AH, Hopkins LN. Asymptomatic carotid stenosis - the not-so-silent disease: changing perspectives from thromboembolism to cognition. J Am Coll Cardiol 2013; 61: 2510-3.

77. Grunwald IQ, Supprian T, Politi M, et al. Cognitive changes after carotid artery stenting. Neuroradiology 2006; 48: 319-23. 
78. Cheng Y, Wang YJ, Yan JC, et al. Effects of carotid artery stenting on cognitive function in patients with mild cognitive impairment and carotid stenosis. Exp Ther Med 2013; 5: 1019-24.

79. Grunwald IQ, Papanagiotou P, Reith W, et al. Influence of carotid artery stenting on cognitive function. Neuroradiology 2010; 52: 61-6.

80. Antonopoulos CN, Kakisis JD, Sfyroeras GS, et al. The impact of carotid artery stenting on cognitive function in patients with extracranial carotid artery stenosis. Ann Vasc Surg 2015; 29: 457-69.

81. Watanabe J, Ogata T, Higashi T, Inoue T. Cognitive change 1 year after CEA or CAS compared with medication. J Stroke Cerebrovasc Dis 2017; 26: 1297-305.

82. Hitchner E, Baughman BD, Soman S, et al. Microembolization is associated with transient cognitive decline in patients undergoing carotid interventions. J Vasc Surg 2016; 64: 1719-25.

83. Zhou W, Baughman BD, Soman S, et al. Volume of subclinical embolic infarct correlates to long-term cognitive changes after carotid revascularization. J Vasc Surg 2017; 65: 686-94.

84. Ruffino MA, Rabbia C. New ischemic brain lesions on DW-MRI after CAS with double-layer stents. Data presented at Leipzig Interventional Course (LINC) 2016. Available at: https:// linc2016.cncptdlx.com/media/1036_Maria_Antonella_Ruffino_26_01_2016_Room_3_Technical_Forum_v2.pdf(accessed 12 June 2017).

85. Musialek P, Bijuklic K, Kolvenbach R, et al. Twelve-month Clinical and Duplex Ultrasound Outcome From the CGuard ${ }^{m}$ CARENET Prospective Multicenter Trial: CARotid Embolic Prevention using MicroNET-Covered Stent System in Patients with Symptomatic and Asymptomatic Carotid Artery Stenosis (manuscript at review; data presented at LINC 2017 in: Musialek P. CGuard ${ }^{\mathrm{TM}}$ MicroNET-covered embolic prevention system routine use in carotid revascularization for stroke prevention: Accumulating clinical evidence. Available athttps://linc2017.cncptdlx.com/media/1300_Piotr_Musialek 26_01_2017_Room_3_-_Technical_Forum.pdf; accessed 12 June 2017).

86. Musialek P, Mazurek A, Trystula M, et al. Durability of stroke prevention using a dual-layer stent system in carotid revascularization in symptomatic and increased-stroke-risk asymptomatic patients: 12-month evidence from the PARADIGM study - ESC 2017 Best Poster. (Abstract) Eur Hear J (Suppl) 2017 (in press).

87. Roffi M, Musialek P. EuroPCR 2017 Highlights: Peripheral Interventions. PCR TV. Available at https://www.youtube.com/ watch? $v=Y l 16 r c F Y d H s$ (accessed 12 June 2017).

88. Fröbert O, Lagerqvist B, Olivecrona GK, et al. Thrombus aspiration during ST-segment elevation myocardial infarction. N Engl J Med 2013; 369: 1587-97.

89. Musialek P. TASTE-less endpoint of 30-day mortality (and some other issues with TASTE) in evaluating the effectiveness of thrombus aspiration in STEMI: Not the "evidence" to change the current practice of routine consideration of manual thrombus extraction. Kardiol Pol 2014; 72: 479-87.

90. Steg PG, James SK, Atar D, et al. ESC Guidelines for the management of acute myocardial infarction in patients presenting with ST-segment elevation. Eur Heart J 2012; 33: 2569-619.

91. Windecker S, Kolh P, Alfonso F, et al. 2014 ESC/EACTS Guidelines on myocardial revascularization. Eur Heart J 2014; 35: 2541619.
92. Serruys PW, Silber S, Garg S, et al. Comparison of zotarolimus-eluting and everolimus-eluting coronary stents. N Engl J Med 2010; 363: 136-46.

93. Paraskevas KI, Veith FJ. Techniques and innovations to improve carotid artery stenting outcomes. Int J Cardiol 2016; 222: 986-7.

94. Musialek P, Pieniazek P. Restenosis after carotid artery stenting versus endarterectomy: the jury is still out! J Endovasc Ther 2010; 17: 271-2.

95. Musialek P, Tracz W, Tekieli L, et al. Multimarker approach in discriminating patients with symptomatic and asymptomatic atherosclerotic carotid artery stenosis. J Clin Neurol 2013; 9: 165-75.

96. Heck DV, Roubin GS, Rosenfield KG, et al. Asymptomatic carotid stenosis: medicine alone or combined with carotid revascularization. Neurology 2017; 88: 2061-5.

97. Paraskevas KI, Veith FJ. Transcervical access, reversal of flow and mesh-covered stents: new options in the armamentarium of carotid artery stenting. World J Cardiol 2017; 9: 416-21.

98. Naylor AR. SPACE: not the final frontier. Lancet 2006; 368: 1215-6.

99. Eckstein HH, Reiff T, Ringleb P, et al. SPACE-2: a missed opportunity to compare carotid endarterectomy, carotid stenting, and best medical treatment in patients with asymptomatic carotid stenoses. Eur J Vasc Endovasc Surg 2016; 51: 761-5.

100. Cleveland TJ, Gaines PA, Venables GS. Carotid artery stenosis: patients should have access to all treatments. BMJ 2010; 340: c1467.

101. Hornung M, Bertog SC, Franke J, et al. Evaluation of proximal protection devices during carotid artery stenting as the first choice for embolic protection. Eurolntervention 2015; 10: 1362-7. 